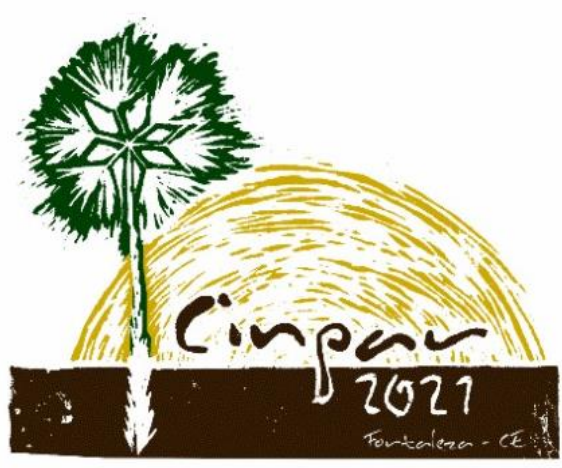

XVII Congresso Internacional sobre Patologia e

Reabilitação das Construções

XVII Congreso Internacional sobre Patología y Rehabilitación de las Construcciones

XVII International Conference on Pathology and Constructions Rehabilitation

FORTALEZA (Brasil), 3 a 5 de junho de 2021

https://doi.org/10.4322/CINPAR.2021.049

\title{
Sinergia dos agentes higrotérmicos na degradação de fachadas
}

\section{Synergy of hygrothermal agents in façade degradation}

\author{
Daiane Teodoro de ANDRADE ${ }^{1}$, Thais Isabela Simão KARDEC ${ }^{2}$, Elton BAUER ${ }^{3}$ \\ ${ }^{1}$ Universidade de Brasília, Brasília, Brasil, daianeteodoro.a@hotmail.com \\ ${ }^{2}$ Universidade de Brasília, Brasília, Brasil, thaiskardec@gmail.com \\ ${ }^{3}$ Universidade de Brasília, Brasília, Brasil, elbauerlem@gmail.com
}

\begin{abstract}
Resumo: Os agentes higrotérmicos, como a temperatura, a chuva dirigida e a radiação solar, afetam diretamente o envelope da edificação. A sua compreensão se mostra necessária no estudo da degradação para melhor avaliação e desempenho da edificação. Busca-se o entendimento de como os agentes higrotérmicos afetam as fachadas favorecendo o aparecimento e a evolução de manifestações patológicas como fissuras e manchas. Através do programa WUFI Pro 5.3 o presente trabalho busca o estudo dos agentes climáticos, chuva dirigida, radiação incidente e consequentemente temperatura superficial e suas respectivas influências na degradação das fachadas, considerando as quatro orientações (norte, sul, leste e oeste), de um edifício localizado em Brasília- DF. Ao correlacionar o índice de incidência de temperatura com o índice de degradação observa-se que há correlação entre os dados, porém há variação entre os resultados de uma fachada para outra, justificando a ocorrência em sinergia dos agentes de degradação, como a chuva dirigida e a incidência de radiação. O maior valor de chuva dirigida $\left(286.1 \mathrm{l} / \mathrm{m}^{2}\right)$ ocorreu na fachada Norte, bem como a maior incidência de choque térmico atenuado $10,9 \%$. Por sua vez a fachada Sul apresenta a menor incidência de chuva dirigida $\left(149.6 \mathrm{l} / \mathrm{m}^{2}\right)$, e as menores incidência de choque térmico pleno e atenuado. Como esperado a fachada Norte apresenta maiores índices de degradação que a fachada Sul, evidenciando a ocorrência sinérgica dos agentes de degradação, como a chuva dirigida e a temperatura.
\end{abstract}

Palavras-chave: WUFI, Fator geral de Danos, Sinergia dos agentes de degradação.

\begin{abstract}
Hygrothermal agents, such as temperature, directed rain and solar radiation, directly affect the building envelope. Its understanding is necessary in the study of degradation for better evaluation and performance of the building. It seeks to understand how hygrothermal agents affect facades favoring the appearance and evolution of pathological manifestations such as fissures and stains. Through the WUFI program $^{\circledast}$ Pro 5.3, the present work seeks the study of climatic agents, directed rain, incident radiation and consequently surface temperature and their respective influences on the degradation of facades, considering the four orientations (north, south, east, and west) of a building located in Brasília- DF. By correlating the temperature incidence index with the degradation index, it is observed that there is a correlation between the data, but there is variation between the results from one façade to another, justifying the synergy occurrence of degradation agents, such as directed rain and the incidence of radiation. The highest directed rainfall value $\left(286.1 \mathrm{l} / \mathrm{m}^{2}\right)$ occurred on the North façade, as well as the highest incidence of attenuated thermal shock $10.9 \%$. In turn, the South façade has the lowest incidence of directed rain $\left(149.6 \mathrm{l} / \mathrm{m}^{2}\right)$, and the lowest incidence of full and attenuated thermal shock. As expected, the North façade has higher rates of degradation than the South façade, evidencing the synergistic occurrence of degradation agents, such as directed rain and temperature.
\end{abstract}

Keywords: WUFI, General Damage Factor, Synergy of degradation agents. 


\section{Introdução}

A degradação dos edifícios não é uniforme, além de ser influenciada pela intensidade dos agentes de degradação, as edificações são compostas por diversos subsistemas que se degradam em diferentes taxas e possuem elementos e componentes, com características e durabilidades distintas (SILVA et al. 2016).

Os agentes higrotérmicos são variáveis em função da zona bioclimática e da orientação das fachadas dentre outros aspectos relacionados a localização da edificação, atuando de maneira singular em cada edificação. Logo a compreensão do processo de degradação relaciona-se com a necessidade de identificar estes agentes e o quanto eles influenciam na perda de desempenho da edificação (Henrique, 2017).

Segundo Shohet et al. (1999) os aspectos relacionados à degradação das fachadas são um processo complexo, principalmente pelo grande número de agentes/fatores envolvidos. A ISO 15686-1: 2011 define como fator de degradação, tudo aquilo que ao agir sobre um sistema contribui para reduzir seu desempenho, onde podemos destacar os agentes biológicos, fatores de uso e esforços, fatores atmosféricos, como a radiação solar, água (chuva dirigida), dentre outros. Já a durabilidade está ligada a sensibilidade de cada sistema estrutural aos diferentes agentes/fatores de degradação, dependente do tipo de ação e mecanismos envolvidos. Em contrapartida não há critérios e requisitos suficientes para um completo entendimento dos agentes higrotérmicos e sua sinergia ao longo da vida útil das edificações.

Segundo Zanoni (2015) a degradação das fachadas é influenciada pelos resultados de amplitude térmica, correlacionada com a absortância das amostras em análise, sendo que a fachada Norte seguida da Fachada Oeste recebe a maior quantidade de radiação solar e chuva dirigida. Em conformidade com Bauer et al. (2015) que demostrou que o fator de danos se mostrou mais elevado nas fachadas Norte e Oeste em Brasília. Portanto, o presente trabalho, objetiva o estudo dos agentes climáticos e suas influências na degradação das fachadas de um edifício localizado em Brasília-DF. Realizou-se simulações através do programa WUFI Pro 5.3 para verificar os níveis de incidência da chuva dirigida, radiação incidente e temperatura na superfície das fachadas. Para critério de comparação calcula-se o índice de incidência de temperatura e correlaciona-o com o indicador de degradação, o qual foi calculado seguindo o método de mensuração da degradação MMD.

\section{Método de Mensuração da degradação}

O método de mensuração da degradação (MMD) estuda as anomalias e as regiões degradadas. Para padronização é realizado a captura fotográfica e ortogonalização de imagens digitais das fachadas, para que as anomalias sejam definidas e quantificadas em escala para cada amostra (BAUER et al. 2020).

Observa-se na figura 1 a metodologia utilizada para mensuração da degradação de fachadas, a qual é dividida em amostras e posteriormente é realizada a sobreposição de uma malha quadriculada em escala de $0,50 \mathrm{~cm}$ por $0,50 \mathrm{~cm}$, que permite a contagem dos danos em função da área afetada. A separação das fachadas em amostras é importante para critérios de comparação com outras edificações com geometrias distintas. Cada amostra deve apresentar uma área entre 50 e $300 \mathrm{~m}^{2}$, estando sempre no mesmo plano, com limite nas articulações estruturais e mesmo elemento arquitetônico (BAUER et al. 2020).

(a)

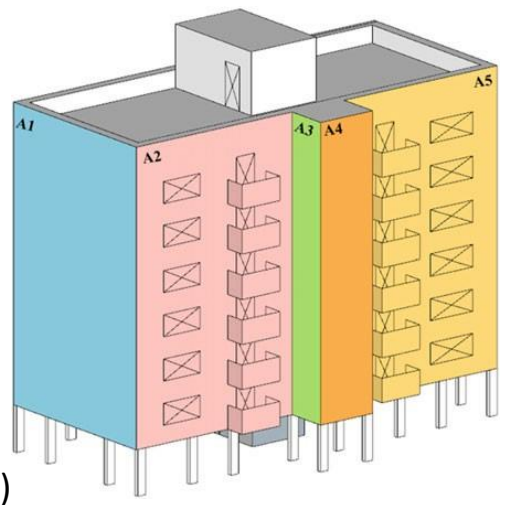

(b)

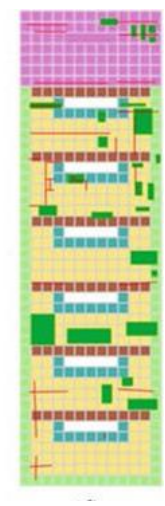

Figura 1- (a) definição das amostras nas fachas; (b) quantificação com sobreposição de malha. (BAUER et al. 2020)

Em conformidade com Bauer et al. (2020) as principais anomalias são classificadas como:

- Descolamento: Todo desprendimento de peça cerâmica independente da causa ou modo de ocorrência.

- Fissuração: Fissuras no revestimento cerâmico independente da sua manifestação e origem.

- Falha nas juntas: Anomalias observadas nas juntas como fissuras ou falhas no selante.

- Manchas: Manchas na superfície de qualquer natureza, como, por exemplo, eflorescências. 
Em estudos realizados por Bauer et al. (2015), ao se analisar uma amostra de 90 fachadas em Brasília-DF, com idade de 5 a 48 anos com objetivo de apresentar uma metodologia de inspeção, identificação, e quantificação das manifestações patológicas, identificou-se que a anomalia mais frequente é o descolamento cerâmico. Seus elevados níveis ocorrem pela influência das tensões térmicas, provenientes da radiação solar, ocasionando dilatações e consequentemente movimentações nas camadas do revestimento, prejudicando a aderência das peças cerâmicas. Em função dos maiores níveis de radiação solar, as fachadas com orientação Norte e Oeste se tornam mais suscetíveis a ocorrência de manifestações patológicas.

Piazzarollo e Bauer (2018) analisaram uma edificação situada no Distrito Federal, utilizando a ferramenta WUFI Pro 5.3, quantificando a temperatura em função da orientação solar. A fachada Norte apresentou os maiores valores de amplitude térmica, sendo a Oeste a menos critica. Os resultados de variação de temperatura são correlacionados com a intensidade anual de radiação incidente, apresentando sinergia entre a absortância da placa cerâmica e a radiação incidente na fachada e a temperatura do ar externo.

\section{$3 \quad$ FGD (Fator geral de danos)}

O FGD considera o custo de reparo e a gravidade das anomalias em função da área afetada, indicando a severidade da degradação e o estado atual do processo de degradação. O FGD é expresso graficamente por uma curva de degradação que reflete a velocidade em que a edificação perde seu desempenho (Bauer et al. 2020). O cálculo do FGD é realizado conforme equação 1 :

$$
F G D=\sum\left[\frac{A_{n} * K_{n} * K_{c, n}}{A * k}\right]
$$

Onde: FGD - Fator Geral de Danos (\%); An - Área de uma fachada afetada por $\mathrm{n}$ tipos de anomalias $\left(\mathrm{m}^{2}\right) ; \mathrm{kn}$ Nível de condição de $n$ anomalias (de 0,00 a 4,00); $k$ - Constante, equivalente ao nível da pior condição $(4,00)$; $\mathrm{kc}, \mathrm{n}$ - Importância relativa das anomalias; A - Área da amostra de fachada $\left(\mathrm{m}^{2}\right) ; n-(\mathrm{n}=1$ - descolamento cerâmico, $n=2$ - falha nas juntas, $n=3$ - fissuras, $n=4$ - manchas).

As curvas de degradação geradas a partir do FGD se toram mais expressivas e acentuadas em amostras com mais de 30 anos. Ao longo do tempo, as manifestações patológicas se associam, como é o caso das fissuras, que permitem o ingresso de água e outros agentes no sistema, favorecendo processos como o descolamento cerâmico e eflorescências. As curvas de degradação mostram que a determinação precoce de falhas é essencial para evitar níveis de degradação acelerados em idades superiores a 30 anos (Bauer e Silva, 2016).

\section{1 lit e FGD}

Para análise das amplitudes térmicas o lit (índice de intensidade da temperatura) correlaciona a amplitude da temperatura média e sua frequência de ocorrência com a frequência de todas as ocorrências das faixas de estudo, leva-se em consideração os efeitos dos ciclos e os valores de temperaturas alcançados no sistema. O lit quando multiplicado pela idade da edificação (lit total) apresenta correlação com os valores de FGD, por tanto há correlação entre a amplitude térmica com a degradação das fachadas com revestimento cerâmico (PIAZZAROLLO; BAUER, 2018). O lit é calculado conforme equação 2, e o lit total conforme equação 3:

$$
\begin{aligned}
\text { Iit } & =\sum\left(\frac{\Delta T_{\text {médio }} \cdot f_{n}}{f_{\text {total }}}\right) \\
\text { Iit total }_{\text {tol }} & =\left(\sum\left(\frac{\Delta T_{\text {médio }} \cdot f_{n}}{f_{\text {total }}}\right)\right) * i
\end{aligned}
$$

Onde: lit: Índice de intensidade da temperatura $\left({ }^{\circ} \mathrm{C}\right)$; $\Delta$ tmédio: é a amplitude de temperatura média da faixa de ocorrência $\left({ }^{\circ} \mathrm{C}\right)$; Fn: Frequência de ocorrência da faixa $n$ de $\Delta T(n=4)$; Ftotal: Frequência de todas as ocorrências das quatro faixas de estudo (ftotal=365); Faixas de estudo: $3,4-11,5^{\circ} \mathrm{C} ; 11,5-19,6^{\circ} \mathrm{C} ; 19,6-$ $27,7^{\circ} \mathrm{C}$ e $27,7-35,8^{\circ} \mathrm{C}$. (NASCIMENTO, 2016); i: Idade da edificação em estudo;

\section{ASPECTOS METODOLÓGICOS}

\subsection{Campo de estudo}

O edifício em estudo possui 35 anos de idade e está localizado em Brasília-DF, possuindo 6 pavimentos, pilotis e cobertura. $O$ revestimento da edificação é em pastilhas cerâmicas, com duas cores predominantes na fachada Sul, Telha (Cor 1) e Branco Gelo (Cor 2), e nas demais fachadas, apenas a cor Telha (Cor 1). 


\subsection{Processo de simulação}

Para simulação no WUFI, utilizou-se um período de dois anos, entre 01/04/2018 a 31/03/2020, mas para análise, excluiu-se o primeiro ano. $O$ sistema construtivo é adotado por: Face da cerâmica externa; Interface Cerâmica/argamassa; Interface argamassa/bloco; Interface bloco/argamassa interna; Face da argamassa interna. Foram definidas no WUFI posições de monitoramento nas camadas de interface de cada camada, conforme observado na Figura 2 a qual representa a seção transversal do elemento.

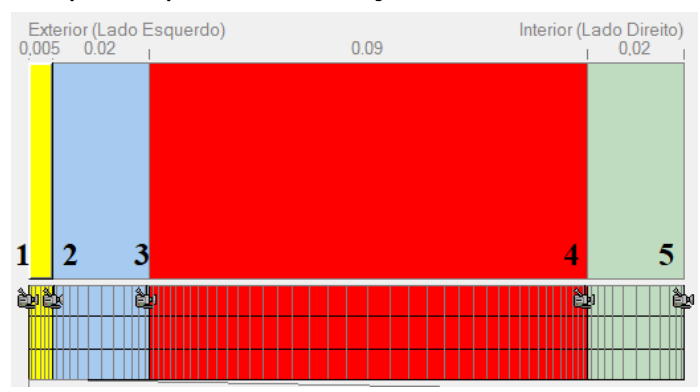

Figura 2 - Corte transversal do elemento construtivo e posições de monitoramento

As propriedades dos materiais foram definidas conforme banco de dados do programa, bem como a refletâncias de $0,2 \%$. Os valores de absortâncias foram obtidos através da tabela fornecida por Dorneles e Roriz (2007), de 63,5\% para cor 1 (telha), e de 39,9\% para cor 2 (branco gelo). Para os dados de clima exterior, foi utilizado o arquivo Typical Meteorological Year (TMY), do Laboratório de Eficiência Energética em Edificações - LABEE da UFSC. Para o clima interno, utilizou-se o estabelecido pela EN 15026. Rodou-se simulações para cada uma das quatro orientações, onde a fachada sul, foi examinada considerando os dados de absortância das duas cores predominantes. Posteriormente foram extraídos os resultados referentes à precipitação (chuva dirigida), incidência de radiação direta e total e temperatura superficial da fachada.

\section{$5 \quad$ Resultados e Discussões}

\subsection{Chuva dirigida anual}

Os resultados da chuva dirigida mostraram a precipitação incidente em cada fachada no decorrer do ano. Obteve-se um total de 286.1, 149.6, 182.8, $179.8\left(\mathrm{l} / \mathrm{m}^{2}\right)$, para respectivamente as fachadas Norte, Sul, Leste e Oeste. A maior incidência de chuva dirigida ocorre na fachada Norte, seguida pelas fachadas Leste e Oeste.

\subsection{Radiação solar}

A Figura 3 apresenta os valores de radiação solar total e direta obtidos pela simulação, ao longo do ano para cada orientação. Observa-se que a radiação solar total e direta na fachada Norte é maior nos meses de abril a setembro, ocorrendo queda da mesma até fevereiro e apresentando um crescente em março. A radiação em Sul se mantem constante e inferior às demais fachadas no decorrer de grande parte do ano e com um crescente entre outubro à janeiro. As fachadas Leste e Oeste apresentam comportamento similar entre elas, porém em agosto a orientação Oeste apresenta um pequeno pico e em janeiro Leste é levemente superior.

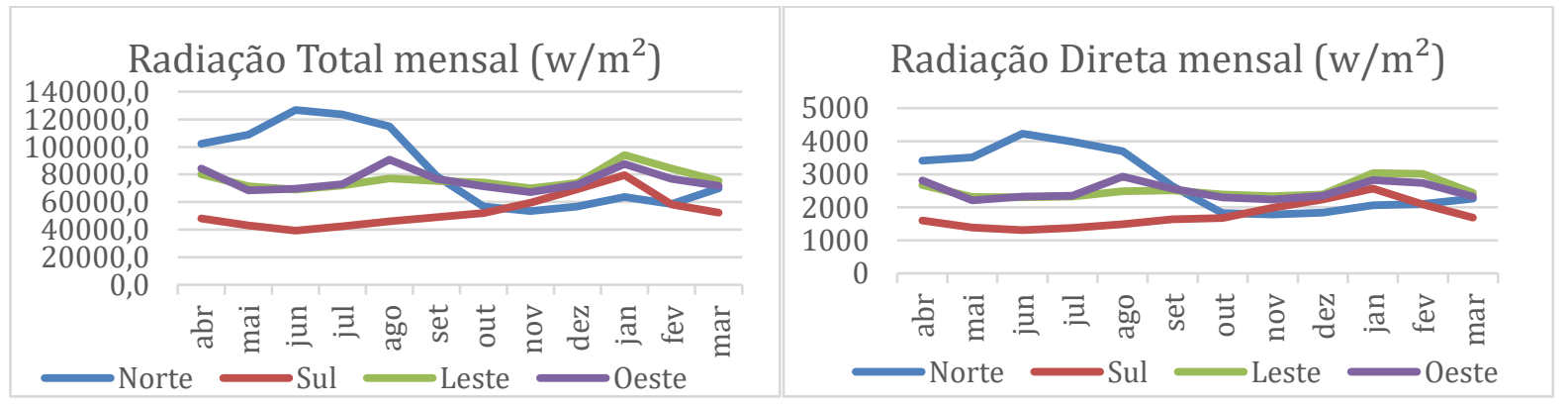

Figura 3 - Radiação total e direta mensal

\subsection{Temperatura máxima e de maior ocorrência}

Os resultados obtidos na simulação computacional, para temperaturas superficiais no revestimento cerâmico, nas quatro fachadas, podem ser observados na figura 4 em forma de Diagramas de Pareto (histogramas com frequências em ordem decrescente da esquerda para direita). 

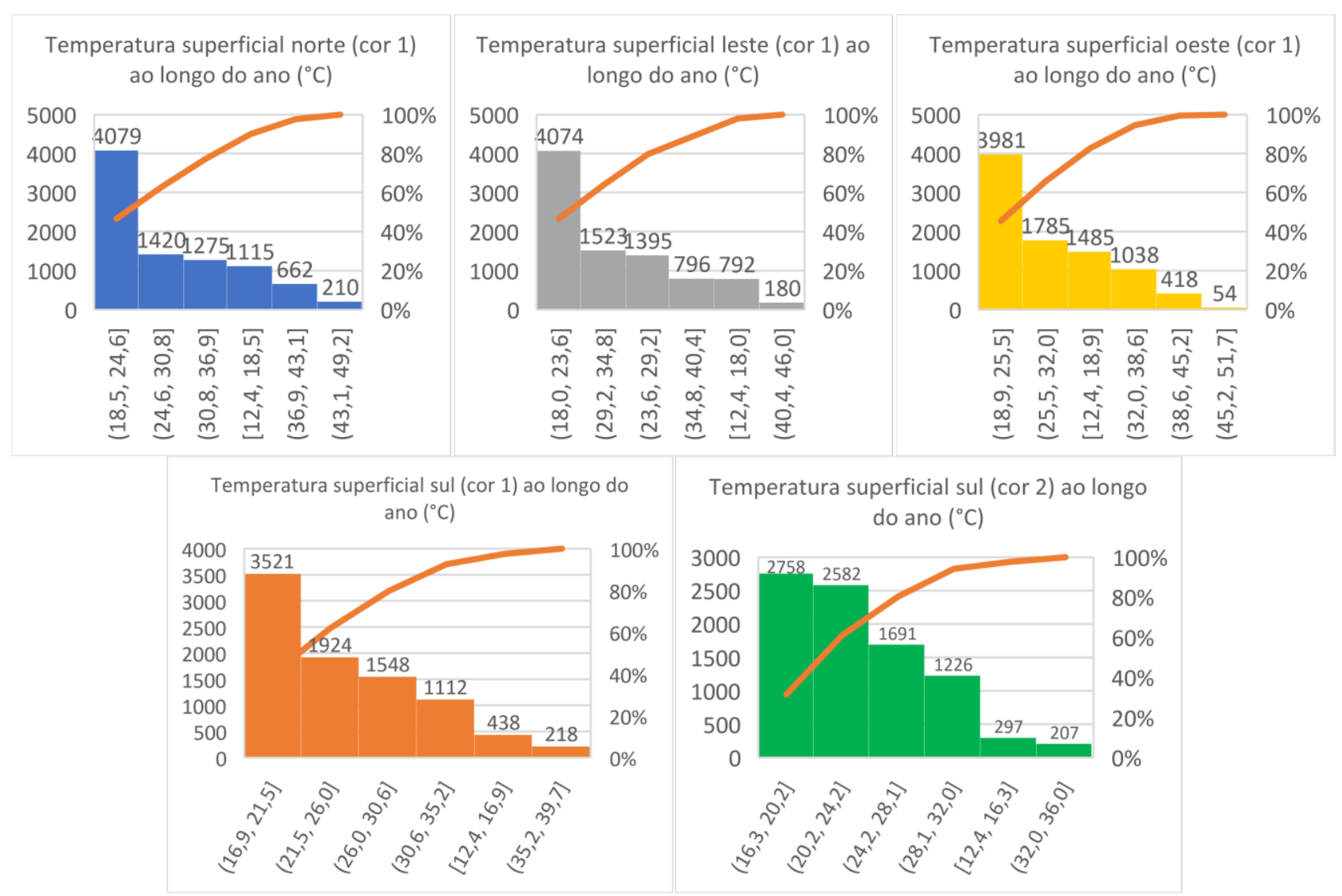

Figura 4- Histogramas das temperaturas superficiais nas fachadas

Com exceção da fachada Sul, as demais fachadas apresentaram faixas de ocorrência de temperaturas da superfície da placa cerâmica entre 18,0 e $25,5^{\circ} \mathrm{C}$. Para a fachada sul, as temperaturas de maiores ocorrências mantiveram-se entre 16,3 e $21,5^{\circ} \mathrm{C}$. O resumo dos resultados obtidos pode ser verificado na Tabela 1.

Tabela 1 - Faixas máximas e de maior ocorrência para as fachadas analisadas

\begin{tabular}{c|cccc}
\hline Fachada & Faixa máxima ${ }^{\circ} \mathbf{C}$ & Ocorrências (\%) & Maior ocorrência ${ }^{\circ} \mathbf{C}$ & Ocorrência (\%) \\
\hline Norte- cor 1 & $43,1-49,2$ & 2,40 & $18,5-24,6$ & 46,60 \\
\hline Sul - cor 1 & $35,2-39,7$ & 2,50 & $16,9-21,5$ & 40,20 \\
\hline Sul - cor 2 & $32,0-36,0$ & 2,40 & $16,3-20,2$ & 31,50 \\
\hline Leste- cor 1 & $40,4-46,0$ & 2,00 & $18,0-23,6$ & 46,50 \\
\hline Oeste- cor 1 & $45,2-51,7$ & 0,60 & $18,9-25,5$ & 45,40 \\
\hline
\end{tabular}

Com o objetivo visualizar o comportamento térmico das fachadas com revestimento cerâmico, plotou-se na Figura 5 os valores máximos de temperatura superficial. A fachada norte apresenta maiores temperaturas entre abril e agosto. A fachada Leste e Oeste, apresentam comportamentos semelhantes, porém, observase uma maior temperatura na fachada Oeste, devido a ocorrência de maiores temperaturas na parte da tarde, momento em que o sol é mais incidente na mesma. A fachada sul cor 2 apresenta os menores resultados, justificado pela sua menor absortância.

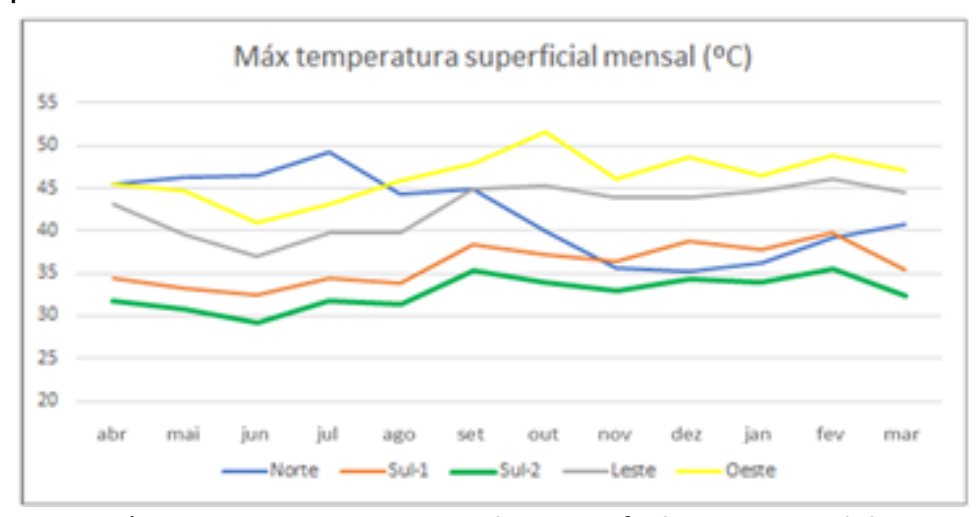

Figura 5 - Máxima temperatura mensal para as fachas norte, sul, leste e oeste 


\subsection{Choque térmico pleno e atenuado}

Considera-se neste trabalho como choque térmico quando há variação maior ou igual a $8^{\circ} \mathrm{C}$ na temperatura superficial da cerâmica em uma hora (choque pleno), e em duas horas (choque atenuado). A figura 6 apresenta um resumo geral da quantidade de choque térmico pleno e atenuado para as orientações norte, sul, leste e oeste para a cor 1, e para a orientação sul cor 2.

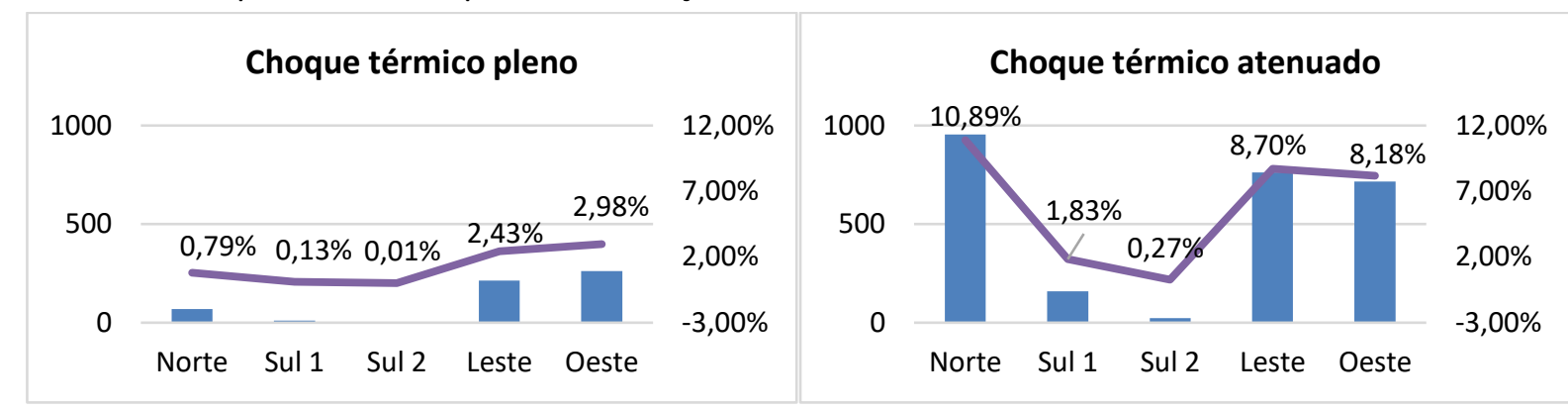

Figura 6- Total de choque térmico pleno e atenuado em cada orientação

Nota-se uma maior ocorrência de choque térmico pleno na orientação Oeste seguido pela Leste. Porém em relação ao choque térmico atenuado a maior ocorrência é em Norte, seguido de Leste e Oeste. A fachada Sul, para ambas as cores, apresenta as menores incidências de choque térmico. Estes resultados condizem com os resultados encontrados por Zanoni (2015) que encontrou como maior incidência 3,5\% de ocorrências de choque térmico pleno na fachada Oeste, o que se justifica pelas temperaturas mais altas no poente e a possível ocorrência de nebulosidade próxima a estes horários.

\subsection{Amplitude térmica superficial}

A amplitude térmica superficial $(\Delta T)$ é a diferença entre a máxima e a mínima temperatura da superfície da fachada durante um dia. Os resultados são observados na figura 7, de maneira análoga ao item 5.3. Em sequência, na Tabela 2, observa-se um resumo dos resultados com as faixas máximas, e as de maior ocorrência, obtidas a partir dos histogramas da figura 7.

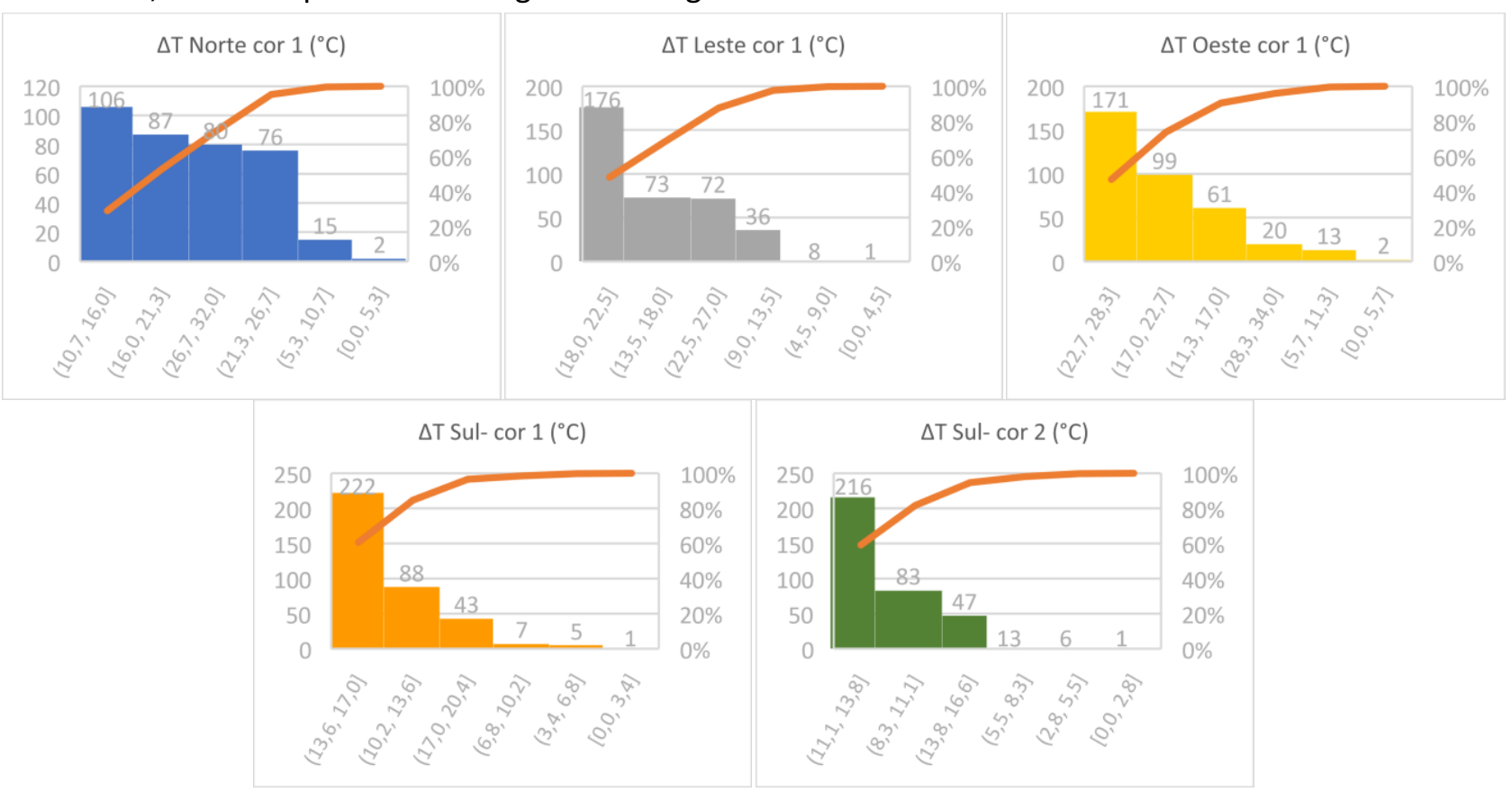

Figura 7- Amplitude térmica superficial diária por orientação

Tabela 2 -Faixas máximas e de maior ocorrência de amplitude térmica diária

\begin{tabular}{c|cccc}
\hline Fachada & Faixa máxima ${ }^{\circ} \mathbf{C}$ & Ocorrências (\%) & Maior ocorrência ${ }^{\circ} \mathbf{C}$ & Ocorrência (\%) \\
\hline Norte- cor 1 & $26,7-32,0$ & 21,86 & $10,7-16,0$ & 28,96 \\
\hline Sul - cor 1 & $17,0-20,4$ & 11,75 & $13,6-17,0$ & 60,66 \\
\hline Sul - cor 2 & $13,8-16,6$ & 12,84 & $11,1-13,8$ & 59,02 \\
\hline Leste- cor 1 & $22,5-27,0$ & 19,67 & $18,0-22,5$ & 48,09 \\
\hline Oeste- cor 2 & $28,3-34,0$ & 5,46 & $22,7-28,3$ & 46,72 \\
\hline
\end{tabular}


Dentre os valores de maior frequência em relação a amplitude da temperatura, a fachada Sul, para cor 1 e cor 2, apresenta faixas de temperatura entre 13,6 e $17,0{ }^{\circ} \mathrm{C}$ e 11,1 e $13,8^{\circ} \mathrm{C}$, mostrando-se mais uniforme que as demais fachadas, em conformidade com os menos valores de choque térmicos para esta orientação.

\subsection{Fator geral de Danos (FGD)}

A Figura 8 apresenta as curvas de degradação padrão e as curvas de degradação obtidas por meio da explanação dos pontos do FGD médio nas orientações norte (cor 1) e sul (cor 1 e 2).

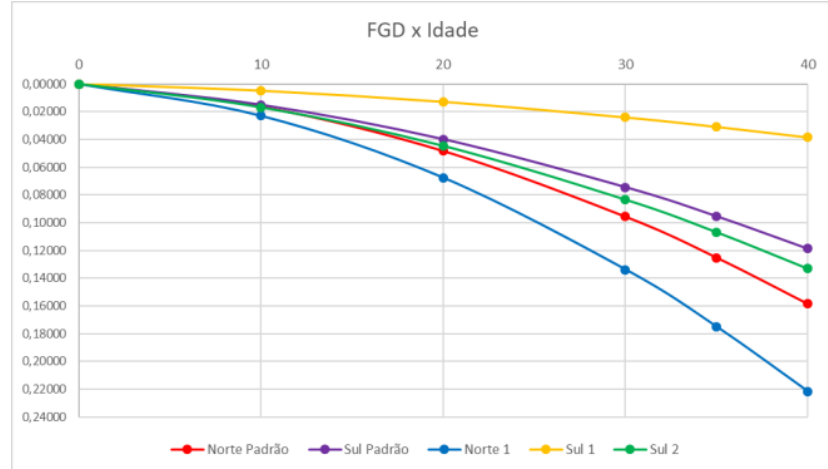

Figura 8- FGD por idade, comparativo das curvas padrões pelos valores do edifício

Nota-se que a curva de degradação da fachada norte do edifício apresentou valores de FGD maiores que a curva padrão. Tal parâmetro induz que a fachada norte do edifício analisado tem maior probabilidade de apresentar sinais de degradação mais avançados que as demais amostras verificadas.

Ao analisarmos as curvas de FGD na orientação sul observamos que a cor 1 apresenta uma menor degradação em relação a curva padrão, enquanto a cor 2 apresenta maiores indicadores de degradação quando comparado com a curva padrão. $O$ que contradiz os resultados apresentados anteriormente, os quais mostram que a cor 2, por possuir uma menor absortância, apresenta as menores amplitudes superficiais e consequentemente uma menor quantidade de choque térmico em relação a cor 1.

Um dos fatores que podem influenciar esse resultado é a geometria da edificação. A existência de proeminências frontais faz com que a cor 1 receba na prática uma menor incidência solar (radiação direta) resultando em menores amplitudes superficiais e uma menor quantidade de choque térmico. Outro fator passível de consideração são as propriedades físicas e químicas das duas cerâmicas, as quais não são conhecidas, e podem apresentar diferentes valores de absorção, resistência ao manchamento etc., decorrentes dos processos de fabricação.

\subsection{1 lit e FGD}

O lit e o lit total foram calculados conforme item 3.1, realizou-se o cruzamento dos resultados com o FGD para as fachadas Norte e Sul, conforme observa-se na figura 9.

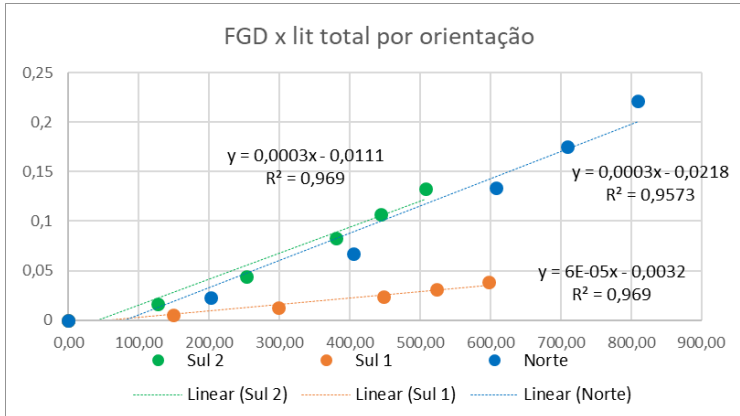

Figura 9- FGD x lit total por orientação

O lit considera os valores de amplitude térmica, sendo estes influenciados pela radiação solar incidente e pela absortância da pastilha cerâmica. Nota-se a semelhança entre o coeficiente angular das retas sul 2 e norte, ambas apresentam a mesma sensibilidade a degradação pela temperatura. Conforme esperado, devido aos resultados discutidos no tópico anterior, a cerâmica 1 em sul apresenta uma sensibilidade a degradação pela temperatura inferior as demais. Os maiores valores de lit total para a fachada norte são justificados pelos maiores valores de incidência de radiação solar que a fachada em sul, o que gera temperaturas mais altas e maiores variações térmicas no decorrer do dia. A comparação entre o lit total e o FGD mostra que os índices são variáveis segundo a orientação das fachadas, evidenciando que diferentes 
níveis de impacto dos agentes higrotérmicos geram diferentes níveis de degradação de maneira correlacionada, corroborando com o resultado da pesquisa realizada por Piazzarollo e Bauer (2018), citada anteriormente, cujos maiores valores do lit foram para as fachas Oeste e Norte.

\section{Conclusões}

- Em relação a radiação solar, a orientação Norte é mais preponderante nos meses de abril a setembro. Já a radiação em Sul se mantem constante e inferior às demais fachadas de Abril a Outubro. As orientações Leste e Oeste apresentam comportamento similares entre si, sem muita variação no decorrer do ano.

- Os resultados de temperatura mostram que a fachada Oeste apresenta faixas de temperaturas máximas maiores que as demais, porém, estas ocorrem com uma menor frequências $(0,60 \%)$. A fachada Sul apresenta os menores valores de temperaturas máximas (32 a 39, $7^{\circ}$ ), principalmente quando utilizada a pastilha cerâmica de cor mais clara, devido às menores absortâncias;

- Quanto a amplitude térmica, a fachada Oeste apresentou as faixas mais altas de temperatura, de 28,3 a $34,0{ }^{\circ} \mathrm{C}$, enquanto fachada sul para a cor 2 , os menores valores de 13,8 a $16,6{ }^{\circ} \mathrm{C}$. Os resultados foram condizentes com valores obtidos de temperatura máxima.

- Ao analisar a ocorrência dos choques térmicos observou-se que a fachada Oeste apresenta as maiores ocorrências de choque térmico pleno (2,98\%). Enquanto para o choque térmico atenuado, a maior ocorrência se dá na fachada Norte $(10,98 \%)$. Sendo que os menores resultados, tanto para o choque térmico pleno quanto para o atenuado, foram obtidos para a fachada em sul para ambas as cores de análise.

- Em relação a chuva dirigida a maior incidência ocorreu para fachada Norte $\left(286.1 \mathrm{l} / \mathrm{m}^{2}\right)$, bem como a menor incidência de chuva dirigida ocorreu para a fachada Sul $\left(149.6 \mathrm{l} / \mathrm{m}^{2}\right)$.

- Ao analisar o lit correlacionado com o FGD pode-se confirmar que cerâmica de cor 1 em sul apresenta uma sensibilidade a degradação pela temperatura inferior as demais. E que a cerâmica sul cor 2 e a norte cor 1, apresentam evolução da degradação semelhante, considerando apenas a influência da temperatura.

- Conclui-se que a degradação da fachada está amplamente correlacionada com a sinergia dos agentes higrotérmicos, sendo o programa WUFI PRO eficaz na predição destes agentes, favorecendo os estudos de degradação e vida útil de fachadas. Verifica-se que para a fachada Norte a maioria dos fatores de degradação analisados neste estudo possuem valores mais elevados, como a incidência de radiação solar mais elevada na orientação Norte em relação a orientação Sul na maior parte do ano, ocasionando em maiores ocorrências de amplitude térmica e choque térmico. Da mesma forma, a maior parte da chuva dirigida ocorre na fachada Norte. A sinergia desses agentes condiz com os maiores valores de FGD na fachada Norte.

\section{Referências}

BAUER, E.; CASTRO, E. K.; SILVA, M. N. B.. Estimativa da degradação de fachadas com revestimento cerâmico: estudo de caso de edifícios de Brasília. Cerâmica, [S.L.], v. 61, n. 358, p. 151-159, jun. 2015. Fap UNIFESP (SciELO). http://dx.doi.org/10.1590/0366-69132015613581786.

BAUER, E.; SOUZA, J. S. de; PIAZZAROLLO, C. B.. Application of the Degradation Measurement Method in the Study of Facade Service Life. Building Pathology And Rehabilitation, [S.L.], p. 105-119, 2020. Springer International Publishing. http://dx.doi.org/10.1007/978-3-030-47302-0_5.

BAUER, Elton; SILVA, Maria De Nazaré. DEGRADAÇÃO DE FACHADAS COM REVESTIMENTO CERÂMICO DE EDIFÍCOS DE BRASÍLIA-DF. Anais do Congresso Brasileiro de Patologia das Construções- CBPAT 2016.

DEUTSCHES INSTITUT FÜR NORMUNG. DIN EN 15026: Hygrothermal performance of building components and building elements: assessment of moisture transfer by numerical simulation. Berlin, 2007.

HENRIQUE, Elaine de Sousa. Sensibilidade à degradação das fachadas: Estudo dos aspectos construtivos. Dissertação de Mestrado em Estruturas e Construção Civil. 2017.

ISO/DIS 15686-1: Buildings - Service life planning. Part 1: General principles and framework, International Organization for Standardization, 2011.

PIAZZAROLLO, Carla B. BAUER, Elton. Quantificação dos agentes de degradação e sua associação com a mensuração da degradação - estudo de caso em Brasília Brasil. U.Porto, Construção 2018.

SHOHET,I.M.;ROSENFELD,Y.;PUTERMAN,M.;GILBOA,E. Deterioration patterns for maintenance management - a methodological approach. In: Durability of Building Materials and Components, Ottawa, 1999.

SILVA, A; DE BRITO, J; GASPAR, P.L. Methodologies for service life prediction of buildings: with a focus on façade claddings. Springer, 2016.

ZANONI, V. A. G. Influência dos agentes climáticos de degradação no comportamento higrotérmico de fachadas em Brasília. Tese (Doutorado) Universidade de Brasília, 2015. 293 p 